First, their review of the literature omits a study from our group (Kellner et al, 1992), in which we found that ECT three times a week had a more rapid onset of antidepressant effect than weekly ECT. In this study, 15 geriatric patients were randomly assigned to receive standard ECT either thrice weekly or once weekly. There was no difference in cognitive effects between the two groups.

Secondly, within the last two years, our ECT service has treated six seriously ill patients who have made complete recoveries after either one or two administrations of ECT. Although this is uncommon, it indicates that there is a subset of patients for whom one or very few administrations of ECT are sufficient to induce remission of severe affective illness. The theoretical implications of this phenomenon are quite important. It may indicate that ECT, in certain patients, can induce a 'switch' mechanism. Whether this happens more often in bipolar patients, and whether or not such early dramatic recoveries predispose to early relapse, would be important issues to study further. Any such predisposition would argue for instituting aggressive continuation or maintenance treatments (either further ECT or antidepressant medications).

These clinical observations indicate that there should be no standard prescription for a course of ECT. The practitioner must follow his/her patient's clinical status closely, and make treatment decisions based on observed responses. While most patients will still require 6-12 administrations of ECT, an important minority may be able to recover with substantially fewer treatments, with resultant cost savings and decreased risk of adverse cognitive effects. We agree strongly with Scott \& Whalley that such clinical phenomena are key areas for further research.

Kelliner, C. K., Monrof, R. R., JR, Pritchett, J. T., et al (1992) Weekly ECT in geriatric depression. Convulsive Therapy. 8, 245- 252

Charles H. Kellner

MARK D. BEALE

Institute of Psychiatry JOHN T. PRITCHETT

Medical University of South Carolina

Charleston

South Carolina 29425-0742

$U S A$

\section{Psychosis and cannabis}

SIR: In their case-control study of psychosis and cannabis use, Rolfe et al (BJP, December 1993, 163, 798-801) seem not to have considered the possibility that the association they found between these variables might have been due to psychotic patients using the drug in an attempt to control their symptoms. No doubt a similar link would have been found between psychosis and neuroleptic use, had they looked for it, but we would not conclude that neuroleptics were therefore a cause of psychosis. What this demonstrates is the unwisdom of drawing causal conclusions from cross-sectional data.

\section{Department of Psychiatry \\ Leicester General Hospital \\ Leicester LE5 4PW}

JAMES LINDESAY

\section{Nasal decongestant and psychiatric disturbance}

SIR: Pseudoephedrine - a stereoisomer of ephedrine is a widely used nasal decongestant, available without prescription. As a sympathomimetic, it has the potential to cause psychiatric disturbance, although it is said to have less effect on the central nervous system than ephedrine. Anxiety, restlessness, visual hallucinations and paranoid psychosis have been reported with preparations containing pseudoephedrine, however (Martindale's Pharmacopoeia).

Sultzer \& Cummings (1989) reviewed cases of drug-induced mania and noted that sympathomimetic amines were among the commoner causes. Dalton (1990) described a case where a first presentation of a bipolar illness was provoked by overdose of pseudoephedrine. I wish to report a case in which an episode of mania was precipitated in a patient with late-onset bipolar illness previously well controlled by lithium.

Case report. A 72-year-old woman with bipolar illness presented to the mental health services with a pseudodementia as a consequence of mania. Before this she had suffered a depressive episode treated by her general practitioner. Her deceased brother suffered a bipolar illness. This first episode responded to haloperidol, on which she was maintained at low dose until she suffered a further manic episode four months later. At this point lithium was introduced with good effect, and eventually continued as sole prophylactic. Her compliance was good, and regular serum lithium estimations ran at about $0.6 \mathrm{mmol} / \mathrm{l}$.

Three years later she was found to be overtalkative, irritable, rather grandiose and sleeping poorly. It was noted that for four weeks she had been taking $60 \mathrm{mg}$ pseudoephedrine hydrochloride nightly, as prescribed by her general practitioner for nasal congestion. The pseudoephedrine was stopped - no other changes were made to her medication - and within a fortnight she had returned to an euthymic state.

The lesson here - aside from an observation of the effects of sympathomimetics - is that all drugs, 